\section{Lighting factors affecting the visibility of a moving display*,**}

\author{
RICHARD D. GILSON $\uparrow, \dagger \dagger$ \\ Naval Aerospace Medical Institute, Pensacola, Florida 32512
}

Compensatory tracking performance was shown to be substantially degraded by oscillation of the visual display at both 1.0 and $2.0 \mathrm{~Hz}$. The severity of this decrement was altered significantly by changes in both the color and the intensity of the display illumination. Performance was significantly better with red light illuminating the display at $0.05 \mathrm{~mL}$ than with blue light at the equivalent luminance. This improvement in performance was similar in magnitude to that found for an increase in broad-band illumination of the display where luminance was increased from $1 / 2 \log$ unit below to $1 / 2 \log$ unit above $0.05 \mathrm{~mL}$. Visual mechanisms that may have been responsible for these findings are suggested.

A recent study by Gilson, Benson, and Guedry (1970) investigated the effects of vestibular stimulation on the performance of a compensatory tracking task. During performance of the task, periodic stimulation of the semicircular canals by angular acceleration induced nystagmic eye movements. As the visual display, an aircraft instrument, was fixed relative to the $S$, such eye movements resulted in image movement across the retina which in turn caused blurring of vision and hence degraded tracking performance. Under these conditions, a systematic increase in the luminance of the tracking display from 0.01 to 10.0 fL markedly improved performance without producing a significant change in nystagmus. The same changes in luminance did not significantly affect performance in the absence of vestibular stimulation. Thus, the alteration in tracking performance with display luminance during vestibular stimulation appeared to depend upon a visual process in which an increase in luminance counteracted the blurring effects of image movement on the retina.

A similar phenomenon has been observed previously. Several investigators (Ludvigh, 1949; Miller, 1958; Mackworth \& Kaplan, 1962; Van Nes, 1968) have reported that

*This research was sponsored jointly by the U.S. Army Aeromedical Research Laboratory and the Naval Aerospace Medical Research Laboratory, and supported in part by the Office of Advanced Research and Technology, National Aeronautics and Space Administration.

* * Opinions or conclusions contained in this report are those of the author. They are not to be construed as necessarily reflecting the view or the endorsement of the Departments of the Army or Navy.

†Address: Naval Aerospace Medical Research Laboratory, Naval Aerospace Medical Institute, Naval Aerospace Medical Center, Pensacola, Florida $\mathbf{3 2 5 1 2 .}$

tThe author gratefully acknowledges Ensign Robert H. Elliott's assistance in constructing and setting up experimental equipment and Mr. David J. Gripka's assistance in analyzing these data. visual acuity is impaired by relative motion between the eyes and a test illumination counteracted this impairment. Miller (1958) and Mackworth and Kaplan (1962) further showed, as did Gilson et al (1970), that the effect of illumination was magnified as the speed of the relative motion was increased.

Ludvigh (1949) attributed the impairment to a reduction in the intensity contrast as a result of continual movement of the image on the retina. The effect of increased illumination was simply to reintensify contrast.

It is curious, however, that the most pronounced effects appeared to occur at low illumination levels. Gilson et al (1970) found that equal log-unit changes in display luminance differentially affected visual tracking performance, with the greatest effect occurring between 0.01 and $0.10 \mathrm{fL}$. It is quite possible that at these low levels of illumination, scotopic visual processes were largely involved, whereas at higher levels photopic processes dominated. The underlying causes, then, might not have been intensity contrast alone, but also the relative degree to which scotopic or photopic visual processes were involved.

The present investigation was undertaken to examine this possibility by comparing Ss' performance when a moving display was illuminated by different colored lights. The lights were equated for luminance, but chosen to enhance photopic vision in one condition and scotopic vision in the other.

\section{Subjects \\ METHOD}

Fifteen naval and marine officers between the ages of 21 and 25 years participated in the experiment. All were student aviators who had passed standard Navy flight physical pattern, and that increasing requirements that included uncorrected $20 / 20$ vision without color defects.

\section{Apparatus and Procedure}

A compensatory visual tracking task provided both a direct practical measure of performance and an indirect measure of visual acuity. The tracking apparatus has been described in detail elsewhere (Gilson et al, 1970). Briefly, a complex "forcing function" input deflected the vertical needle of an aircraft-localizer/glideslope indicator while the $\mathbf{S}$ attempted to maintain the needle in a null position by manipulation of a control stick. Deviations of the needle from this position were considered errors, and a voltage proportional to these deviations was electronically integrated over consecutive intervals throughout a trial.

In the present experiment a sinusoidal forcing function with a 14-sec period was used to deflect the vertical needle. Errors were also integrated for $42 \mathrm{sec}$, or three complete cycles of the forcing function, in order to obtain one index of performance for each experimental condition.

Relative motion between the eyes and the display was introduced by moving a mirror view of the display. The $\mathbf{S}$ binocularly viewed the display reflection in a mirror that was oscillated about its vertical axis. One end of the mirror was connected by a jointed arm to an off-center position on a remote shaft. The shaft was rotated by a two-speed constant-speed motor, causing the mirror to move in approximately a sinusoidal manner. With the S's eyes and the display equidistant from the mirror $(40 \mathrm{~cm})$, the visual angular displacement of the image was the same as the angular displacement of the mirror. The result was the effective movement of the display to the left and right of the S's view according to the same sinusoidal function as the mirror.

The visual angle between the two extreme display positions was $10 \mathrm{deg}$. The frequency of display oscillation was $1.0 \mathrm{~Hz}$ with the motor at low speed and $2.0 \mathrm{~Hz}$ with the motor at high speed. These values of angular displacement and frequency are in the range of those obtained for nystagmic responses in the previous study (Gilson et al, 1970).

The $\mathbf{S}$ sat in front of the mirror with his head supported by a chinrest. The control stick was attached within convenient reach to the bottom of the table and was activated from side to side to correspond with needle movements.

The display was illuminated in the otherwise dark room by a Kodak 


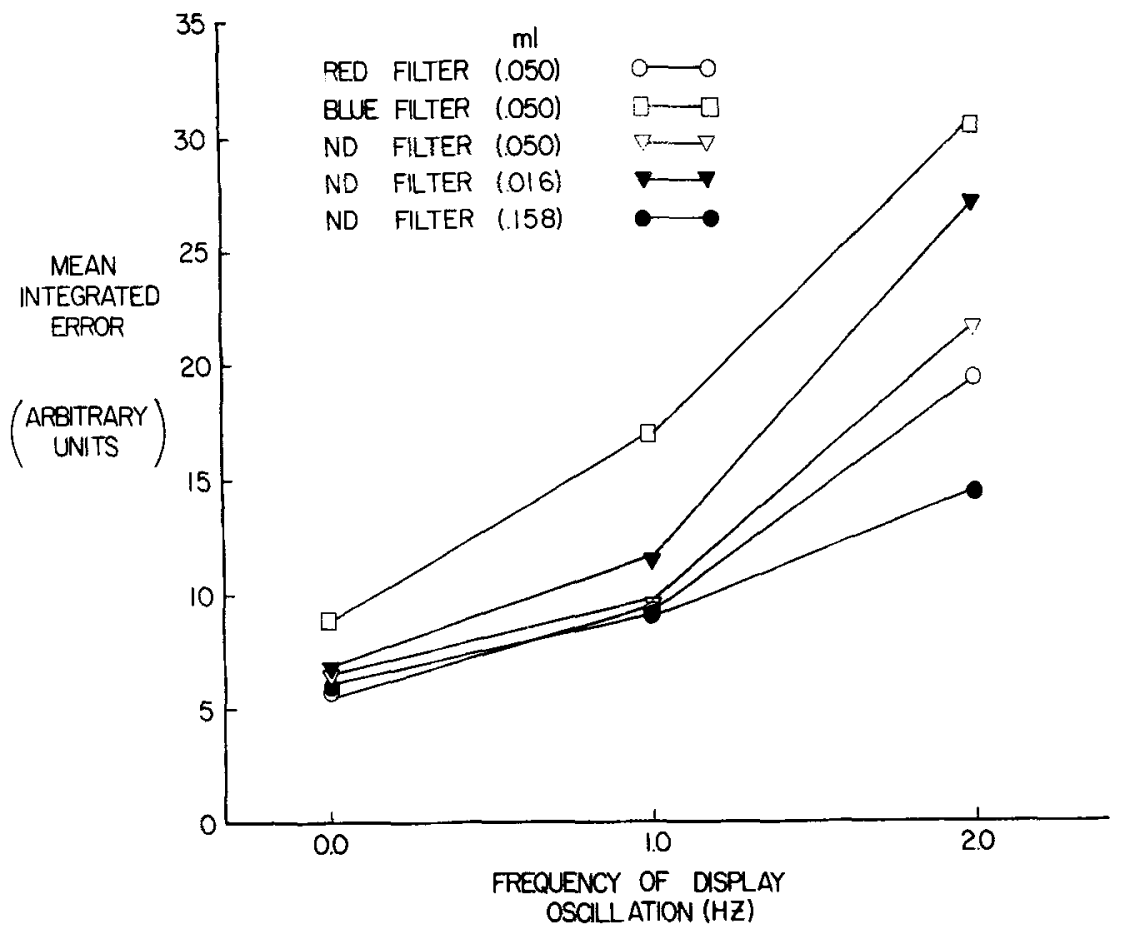

Fig. 1. Effect of display illumination on compensatory tracking performance during sinusoidal oscillation of the display. Each point is the mean integrated error score over $42 \mathrm{sec}$ of tracking for 15 Ss. Error scores are plotted for the condition of illumination and frequency of display oscillation. $\mathrm{ND}=$ neutral density.

Carousel 750 automatic slide projector. Light was projected through a tube to illuminate the display, which was shielded from direct viewing.

Experimental conditions of both wavelength and luminance were controlled by Kodak Wratten gelatin filters mounted in glass slides and inserted into the projector. Two slides contained cutoff filters, No. 47B (blue) and No. 25A (red), that passed energy below approximately 500 millimicrons and above approximately 580 millimicrons, respectively. The blue filter should, according to accepted scotopic and photopic curves (Bartley, 1951), stimulate scotopically with greater efficiency at low illumination than it would photopically. Conversely, the red filter should stimulate photopically with greater efficiency than it would scotopically.

The red, the blue, and a third neutral density filter were equated for luminance by superimposing additional neutral density filters into the slides until the luminance on the display was $0.05 \mathrm{~mL}$. Two other slides also containing neutral density filters were selected to give values of $1 / 2 \log$ unit above and below $0.05 \mathrm{~mL}$ ( 0.158 and $0.016 \mathrm{~mL}$, respectively).

The luminance of the display was measured with the aid of a card sprayed with the same white paint as the display needles. The card was density filter and with the display oscillating at $1.0 \mathrm{~Hz}$. At the end of this practice period, the filter and display frequency were immediately changed to the first condition of the selected sequence. Approximately $15 \mathrm{sec}$ of tracking during each condition were allowed for tracking performance to stabilize before the 42 -sec scoring interval began.

\section{RESULTS AND DISCUSSION}

The results are shown in Fig. 1. It is evident that as frequency of display oscillation was increased, so was tracking error, regardless of the illuminating condition. This finding is in agreement with other studies (Ludvigh \& Miller, 1958; Miller, 1958) where impairment of acuity was positively correlated with increased velocity of the test object.

The type of illumination appears to determine the rate of increase of tracking error with display frequency. As indicated previously, other studies (Miller, 1958; Mackworth \& Kaplan, 1962; Gilson et al, 1970) have shown that increased illumination reduced the acuity loss or decrement in tracking performance produced by either display or eye movements and that this effect increased with the speed of the motion. This is apparent in the present results, even with only a 1-log-unit overall change in luminance. The curves depicting the results for the three neutral density filters diverge, as frequency increases, to a degree where the performance at the brightest illumination is significantly better $(p<.01)$ than at the lowest illumination at $2.0 \mathrm{~Hz}$.

In a similar manner, the curves for tracking performance with the blue and red filters diverge and differ significantly $(p<.01)$ at both 1.0 and $2.0 \mathrm{~Hz}$. Despite equation for luminance, the differences in these curves are similar to those obtained for the log-unit change in luminance between the dim and bright neutral density filters.

Thus, this effect is not merely the result of luminance changes, but of some other factor associated with changes in both luminance and wavelength. The low luminance levels and specific wavelengths for which this effect is most apparent border on the transition interval between scotopic and photopic stimulation (Bartley, 1951). This lends support to the hypothesis that differences between scotopic and photopic visual processes may be involved.

The similarity in performance for the red and equally bright neutral density filter may well be the result of the light passed by the neutral filter stimulating both visual processes, with the more efficient photopic process prevailing. 


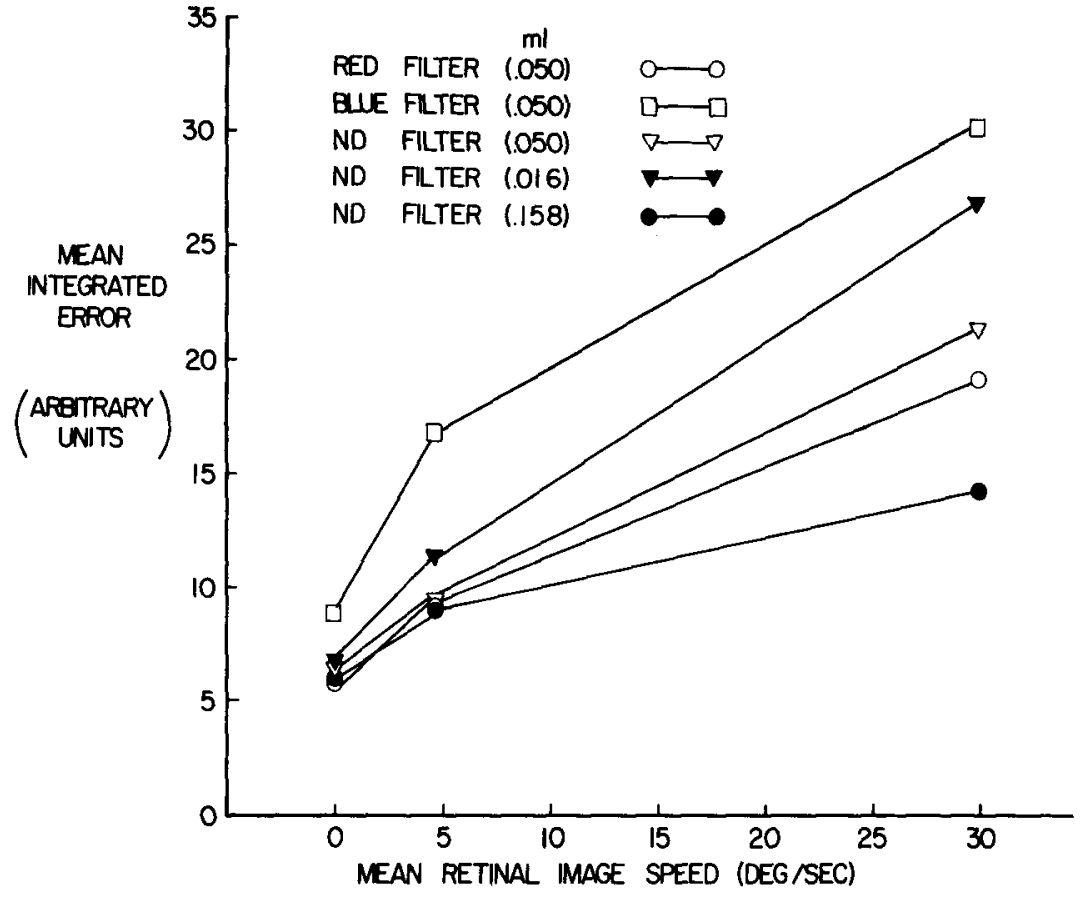

Fig. 2. The same data as shown in Fig. 1 except plotted for the average absolute velocity of the display image passing over the retina of the eye. These values were computed from the differential velocity of the eye and the display. Eye velocities were interpolated from Huddleston (1969).

Graham (1965) has speculated on how movement of an image across the retina may reduce border contrast. His arguments are based in part on the reduced stimulation of a given retinal element according to the Bunsen-Roscoe law and in part on inhibition and facilitation of adjacent retinal elements as the image moves across them. If such processes do in fact govern border contrast during image movement, then the present results indicate that one or both of these processes are different for the rods than for the cones.

Numerous techniques have been used previously to achieve retinal image motion: by moving a test object in either a circular (Ludvigh, 1949), horizontal (Ludvigh \& Miller, 1958), or vertical (Miller, 1958; Van Nes, 1968) path; by rotating the $S$ at constant velocity while he viewed a reflection of a stationary test object from a mirror rotating with him (Miller, 1958); by requiring the $S$ to fixate on a moving fixation target during which time a stationary test object was momentarily presented in the viewing field (Mackworth \& Kaplan, 1962); and by requiring the $S$ to view a display fixed relative to himself, while undergoing vestibular stimulation sufficient to evoke nystagmic eye movements (Guedry, 1968; Gilson et al, 1970).

Except under the latter two affords a more veridical independent variable. The shape of these curves is now similar to that obtained by Mackworth and Kaplan (1962) who, by varying only eye velocity, were presumably measuring retinal image speed more directly.

These results emphasize those of others cited previously (Ludvigh, 1949; Mackworth \& Kaplan, 1962; Miller, 1958; Van Nes, 1968; Gilson et al, 1970) that even a small change in luminance at low levels will strongly affect visual performance when there is relative motion between the eyes and a display. In addition, this process appears to be similarly influenced by changes in spectral distribution at low but equivalent luminance levels and is apparently independent of the cause of the relative motion.

\section{REFERENCES}

BARTLEY, H. S. The psychophysiology of vision. In S. S. Stevens (Ed.), Handbook of experimental psychology. New York: Wiley, 1951. Pp. 921-984.

GILSON, R. D., BENSON, A. J., \& GUEDRY, F. E. Influence of vestibular stimulation and display luminance on the performance of a compensatory tracking task. Aerospace Medicine, 1970, 41 . 1231-1237.

GRAHAM, C. H. Perception of movement. In C. H. Graham (Ed.), Vision and visual perception. New York: Wiley, 1965. Pp. 575-588.

GUEDRY, F. E. Relations between vestibular nystagmus and visual performance. Aerospace Medicine, 1968. 39, 570-579.

HUDDLESTON, H. F. Oculomotor pursuit of vertical sinusoidal targets. Nature, 1969, 222, 572-574.

LUDVIGH, E. J. Visual acuity while one is viewing a moving object. Archives of Ophthalmology, 1949, 42, 14-22.

LUDVIGH, E. J., \& MILLER, J. W. Study of visual acuity during the ocular pursuit of moving test objects. I. Introduction. Journal of the Optical Society of America, 1958, 48, 799-802.

MACKWORTH, N. H., \& KAPLAN, I. T Visual acuity when eyes are pursuing moving targets. Science, 1962, 136, 387-388.

MILLER, J. W. Study of visual acuity during the ocular pursuit of moving test objects. II. Effects of direction of movement, relative movement, and illumination. Journal of the Optical Society of America, $1958,48,803-808$.

VAN NES, F. L. Enhanced visibility by regular motion of retinal image. American Journal of Psychology, 1968, 81 367-374.

NOTE

1. A pilot experiment for the present study utilizing electrooculographic recordings from one $S$ did not reveal any differences in oculomotor pursuit for any of the lighting conditions at display frequencies of either 1.0 or $2.0 \mathrm{~Hz}$, yet clearly showed the sinusoidal eye movements.

(Accepted for publication May 11, 1971.) 